\title{
A Resilient Approach for Distributed MPC-Based Economic Dispatch in Interconnected Microgrids
}

\author{
Wicak Ananduta, José María Maestre, Carlos Ocampo-Martinez, and Hideaki Ishii
}

\begin{abstract}
Economic dispatch of interconnected microgrids that is based on distributed model predictive control (DMPC) requires the cooperation of all agents (microgrids). This paper discusses the case in which some of the agents might not comply with the decisions computed by performing a DMPC algorithm. In this regard, these agents could obtain a better performance at the cost of degrading the performance of the network as a whole. A resilient distributed method that can deal with such issues is proposed and studied in this paper. The method consists of two parts. The first part is to ensure that the decisions obtained from the algorithm are robustly feasible against most of the attacks with high confidence. In this part, we employ a two-step randomization-based approach to obtain a feasible solution with a predefined level of confidence. The second part consists in the identification and mitigation of the adversarial agents, which utilizes hypothesis testing with Bayesian inference and requires each agent to solve a mixed-integer problem to decide the connections with its neighbors. In addition, an analysis of the decisions computed using the stochastic approach and the outcome of the identification and mitigation method is provided. The performance of the proposed approach is also shown through numerical simulations.
\end{abstract}

Index Terms-Economic dispatch, distributed MPC, distributed optimization, resilient algorithm, scenario-based approach

\section{INTRODUCTION}

An application of distributed model predictive control (DMPC) methods that has increasingly gained attention is as a control approach of power networks, particularly to solve economic dispatch problems [1]-[3]. DMPC methods are perceived to be suitable for a power network, which is of large-scale nature, especially when there exist distributed generation and storage units. In a distributed setting, there exist a number local controllers, responsible to control a part of the system and can communicate among each other. Therefore, the computational burden to compute the control inputs, which might be quite high, can be distributed. Furthermore, a distributed scheme is also scalable and can deal with failures better than the centralized counterparts

W. Ananduta and C. Ocampo-Martinez are with Institut de Robòtica i Informàtica Industrial, CSIC-UPC, Barcelona, Spain (emails: \{wananduta, cocampo\}eiri.upc.edu).

J. M. Maestre and H. Ishii are with Department of Computer Science, Tokyo Institute of Technology, Yokohama, Japan (emails: pepemaestre@us.es, ishiidc.titech.ac.jp).

J. M. Maestre is also with Department of System and Automation Engineering, University of Seville, Seville, Spain.

This work has received funding from the European Union's Horizon 2020 research and innovation programme under the Marie Skłodowska-Curie grant agreement No 675318 (INCITE) and is also supported by the Spanish State Research Agency through the María de Maeztu Seal of Excellence to IRI (MDM-2016-0656). Financial support by the Spanish MINECO project DPI2017-86918-R and the Japanese Society for the Promotion of Science (scholarship PE16048) is also gratefully acknowledged.
[4]. For an economic dispatch problem of a power network, a distributed scheme can be implemented by decomposing the network into a group of interconnected microgrids [5]. Then, a distributed optimization algorithm, e.g., [2], [3], can be employed to solve the optimization problem behind the DMPC scheme. In this regard, generally, a DMPC strategy requires cooperation of all agents (microgrids), i.e., all agents must agree to perform the algorithm accordingly and comply with the obtained decision.

This paper discusses a cooperation issue of DMPC strategies for an economic dispatch, in which some agents in the network, denoted by adversarial agents, do not always implement the control inputs obtained from the distributed algorithm. By performing such actions, adversarial agents can perform better at the expenses of the performance of the other agents. In order to tackle this issue, we develop a combination of passive and active methodologies, which is introduced in [6]. As a passive method, we formulate the dispatch problem such that the computed decision is robust with respect to the adversarial actions. To that end, in [6], we consider the adversarial actions (attacks) as bounded disturbances and formulate a robust program to ensure the feasibility of the solutions in the presence of the attacks. On the other hand, the active method identifies the adversarial agents and blocks the attacks. The identification approach consists in implementing the hypothesis testing based on Bayesian inference. Furthermore, each agent must also solve a local mixed integer programming problem to decide the connection with the neighbors.

In this paper, we develop further our methodology by considering a stochastic approach [7] as the passive method such that we do not need to assume that the bounds of the attacks and system disturbances are known. The stochastic approach, explained in Section III, consists of two steps. The first step is to compute the probabilistic bounds of the disturbances using a scenario-based program. In the second step, a robust program is formulated using the computed probabilistic bounds. Furthermore, the probabilistic bounds are used in the active scheme to detect an adversarial action. Hence, the main contribution of this paper is the improvement of the methodology that we propose to deal with the compliance issue for DMPC strategies applied to the economic dispatch problem. Furthermore, in Section V, we characterize the computed control inputs obtained by the proposed approach and we show analytically how the active method is able to identify the adversarial agents in the network. Additionally, a numerical study is also carried out to show the performance of the proposed approach. 
Notations: The sets of real numbers and integers are denoted by $\mathbb{R}$ and $\mathbb{Z}$, respectively. Moreover, for any $a \in \mathbb{R}$, $\mathbb{R}_{\geq a}$ denotes the subset of $\mathbb{R}$ that is defined by $\{b: b \geq$ $a, b \in \mathbb{R}\}$. A similar definition is used for $\mathbb{Z}_{>a}$ and the strict inequality cases. The vector $\mathbb{1}_{n}$ denotes $\left[\begin{array}{llll}1 & 1 & \cdots & 1\end{array}\right]^{\top} \in \mathbb{R}^{n}$. The set cardinality and Euclidean norm are denoted by $|\cdot|$ and $\|\cdot\|_{2}$, respectively. Supposing that $\Omega_{i}$, for $i=1,2, \ldots, n$, is a subset of $\mathbb{R}^{n_{i}}$, then the Cartesian product over the sets $\Omega_{i}$ is defined by $\prod_{i=1}^{n} \Omega_{i}=\Omega_{1} \times \ldots \times \Omega_{n}$. Furthermore, $\mathbb{P}(\cdot)$ denotes the probability measure, and $\mathbb{P}(\cdot \mid \cdot)$ denotes the conditional probability measure. Finally, discrete-time instants are denoted by the index $k$.

\section{Distributed Energy Management And ADVERSARY MODEL}

\section{A. Dynamic Economic Dispatch Problem}

Let the network of microgrids be described by an undirected graph $\mathcal{G}=(\mathcal{N}, \mathcal{E})$, where $\mathcal{N}$ denotes the set of microgrids and $\mathcal{E}$ denotes the set of edges, which represents the physical interconnection of the microgrids. Furthermore, denote the set of neighbors of microgrid $i$ by $\mathcal{N}_{i}=\{j \in$ $\mathcal{N}:(i, j) \in \mathcal{E}\}$. We consider that each microgrid $i \in \mathcal{N}$ consists of a dispatchable generation unit, a non-dispatchable generation unit, a storage unit, and an aggregated load. Moreover, each microgrid can operate in the grid-connected and island modes. Furthermore, two connected microgrids $i$ and $j$, i.e., $(i, j) \in \mathcal{E}$, can also transfer power, and microgrids that are connected to the main grid can also import power from it. We follow a similar formulation of the dynamic economic dispatch problem as in [6], but we will not consider any assumption on the bounds of the uncertain variables.

Firstly, let the decision variables of each microgrid $i \in \mathcal{N}$ be defined by $p_{i, k}^{\mathrm{g}}, p_{i, k}^{\mathrm{st}}, p_{i, k}^{\mathrm{im}}, p_{j i, k}^{\mathrm{t}} \in \mathbb{R}$, which denote the total power generated by the dispatchable units, the power delivered by/to the storage, the imported power from the main grid, and the power transferred between microgrids $i$ and $j$, respectively. Secondly, let the dynamics of the storage unit in microgrid $i$ be represented as follows:

$$
x_{i, k+1}=a_{i} x_{i, k}+b_{i} p_{i, k}^{\mathrm{st}},
$$

where $x_{i, k}$ denotes the state of charge (SoC) of the storage unit, $a_{i} \in(0,1]$ denotes its efficiency and $b_{i}=-\frac{T_{\mathrm{s}}}{e_{\mathrm{cap}, i}}$, where $T_{\mathrm{s}}$ and $e_{\mathrm{cap}, i}$ denote the sampling period and the maximum capacity of the storage unit, respectively. Moreover, consider the operational constraints of the components of microgrid $i$ as follows:

$$
\begin{aligned}
x_{i}^{\min } & \leq x_{i, k} \leq x_{i}^{\max }, \quad-p_{i}^{\mathrm{ch}} \leq p_{i, k}^{\mathrm{st}} \leq p_{i}^{\mathrm{dh}}, \\
p_{i}^{\mathrm{g}, \min } & \leq p_{i, k}^{\mathrm{g}} \leq p_{i}^{\mathrm{g}, \max }, \quad 0 \leq p_{i, k}^{\mathrm{im}} \leq p_{i}^{\mathrm{im}, \max }, \\
-p_{i}^{\mathrm{t}, \max } & \leq p_{j i, k}^{\mathrm{t}} \leq p_{i}^{\mathrm{t}, \max }, \quad \forall j \in \mathcal{N}_{i},
\end{aligned}
$$

where $x_{i}^{\min }, x_{i}^{\max } \in[0,1]$ denote the minimum and the maximum SoC of the storage unit, respectively; $p_{i}^{\text {ch }} \in \mathbb{R}_{\geq 0}$ and $p_{i}^{\mathrm{dh}} \in \mathbb{R}_{\geq 0}$ denote the maximum charging and discharging power of the storage; $p_{i}^{\mathrm{g}, \min }, p_{i}^{\mathrm{g}, \max } \in \mathbb{R}_{\geq 0}$ denote the minimum and the maximum of the total power generated by the DG units of microgrid $i$, respectively; $p_{i}^{\text {im,max }} \in \mathbb{R}_{\geq 0}$ denotes the maximum imported power from the main grid; and $p_{i}^{\mathrm{t}, \max } \in \mathbb{R}_{>0}$ denotes the maximum energy that can be transferred between microgrids $i$ and $j$. Additionally, consider the power balance equations of each microgrid $i \in \mathcal{N}$ as follows:

$$
\begin{aligned}
p_{i, k}^{\mathrm{d}}-p_{i, k}^{\mathrm{st}}-p_{i, k}^{\mathrm{g}}-p_{i, k}^{\mathrm{im}}-\sum_{j \in \mathcal{N}_{i}} p_{j i, k}^{\mathrm{t}} & =0, \\
p_{i j, k}^{\mathrm{t}}+p_{j i, k}^{\mathrm{t}} & =0, \quad \forall j \in \mathcal{N}_{i},
\end{aligned}
$$

where $p_{i, k}^{\mathrm{d}}$ denotes the uncertain power disturbance that represents the difference between the uncertain load and the power generated by the non-dispatchable units.

Finally, collect the decision variables of microgrid $i$ as a vector, denoted by $\boldsymbol{u}_{i, k}=\left[\begin{array}{llll}p_{i, k}^{\mathrm{st}} & p_{i, k}^{\mathrm{g}} & p_{i, k}^{\mathrm{im}} & \boldsymbol{u}_{i, k}^{\mathrm{c} T}\end{array}\right]^{\top}$, where $\boldsymbol{u}_{i, k}^{\mathrm{c}}=\left[p_{j i, k}^{\mathrm{t}}\right]_{j \in \mathcal{N}_{i}}^{\top}$. Based on the preceding model of the system, the dynamic economic dispatch problem behind the DMPC scheme is stated as follows:

$$
\begin{gathered}
\underset{\left\{\left\{\boldsymbol{u}_{i, \ell \mid k}\right\}_{i \in \mathcal{N}}\right\}_{\ell=k}^{k+h_{p}-1}}{\operatorname{minimize}} \sum_{i \in \mathcal{N}} \sum_{\ell=k}^{k+h_{p}-1} J_{i, \ell}\left(\boldsymbol{u}_{i, \ell \mid k}\right) \\
\text { subject to } \quad \boldsymbol{F}_{i} \boldsymbol{u}_{i, \ell \mid k} \leq \boldsymbol{f}_{i, \ell}, \forall i \in \mathcal{N}, \\
\boldsymbol{u}_{i, \ell \mid k}^{\mathrm{c}}+\sum_{j \in \mathcal{N}_{i}} \boldsymbol{G}_{i j} \boldsymbol{u}_{j, \ell \mid k}^{\mathrm{c}}=\mathbf{0}, \forall i \in \mathcal{N},
\end{gathered}
$$

for all $\ell \in\left\{k, \ldots, k+h_{p}-1\right\}$, where $h_{p} \in \mathbb{Z}_{\geq 1}$ denotes the prediction horizon. The stage cost function in (7a) is defined as $J_{i, k}=\boldsymbol{u}_{i, k}^{\top} R_{i} \boldsymbol{u}_{i, k}$, for all $i \in \mathcal{N}$ and $k \in \mathbb{Z}_{\geq 0}$, where $R_{i}=\operatorname{diag}\left(\left[\begin{array}{llll}c_{i}^{\mathrm{st}} & c_{i}^{\mathrm{g}} & c_{i}^{\mathrm{im}} & c_{i}^{\mathrm{t}} \mathbb{1}_{\left|\mathcal{N}_{i}\right|}^{\top}\end{array}\right]\right)>0$, in which $c_{i}^{\mathrm{st}}, c_{i}^{\mathrm{g}}, c_{i}^{\mathrm{im}}, c_{i}^{\mathrm{t}} \in \mathbb{R}_{>0}$ denote the per-unit cost of storage operation, producing energy, buying energy from the main grid, and transferring energy to/from the neighbor due to losses, respectively [1]. Moreover, the local constraints (7b) with the appropriate $\boldsymbol{F}_{i}$ and $\boldsymbol{f}_{i, \ell}$ are constructed from (1)(5) and only depend on local decision variables whereas the coupled constraints (7c) with the appropriate matrices $\boldsymbol{G}_{i j}$, for all $j \in \mathcal{N}_{i}$, are formed from (6).

\section{B. Distributed Optimization Method}

We solve Problem (7) in a distributed manner by assuming bidirectional communication between neighboring agents. Many distributed optimization algorithms can be applied as the DMPC strategy for this problem, e.g., [2], [3]. For clarity of the explanation, we consider the dual-ascent method (Algorithm 1). Since (7b)-(7c) form a polyhedral set and (7a) is strictly convex, the solutions coming from Algorithm 1, denoted by $\boldsymbol{u}_{i, \ell \mid k}^{\star}$, for all $i \in \mathcal{N}$ and $\ell \in\left\{k, \ldots, k+h_{p}-1\right\}$, converge to the optimal solution of Problem (7) [6], [8].

\section{Adversary Model}

It is considered that some of the agents (microgrids) might perform adversarial actions. The adversary model of the system is stated by the following definitions and assumptions.

Definition 1: An agent $i$ is regular if it always implements its control input according to the decision obtained from the DMPC strategy, i.e., $\boldsymbol{u}_{i, k}=\boldsymbol{u}_{i, k \mid k}^{\star}$, for all $k \geq 0$. Otherwise, agent $i$ is adversarial. Furthermore, denote the set of regular agents by $\mathcal{R}$ and that of adversarial agents by $\mathcal{A}$. 


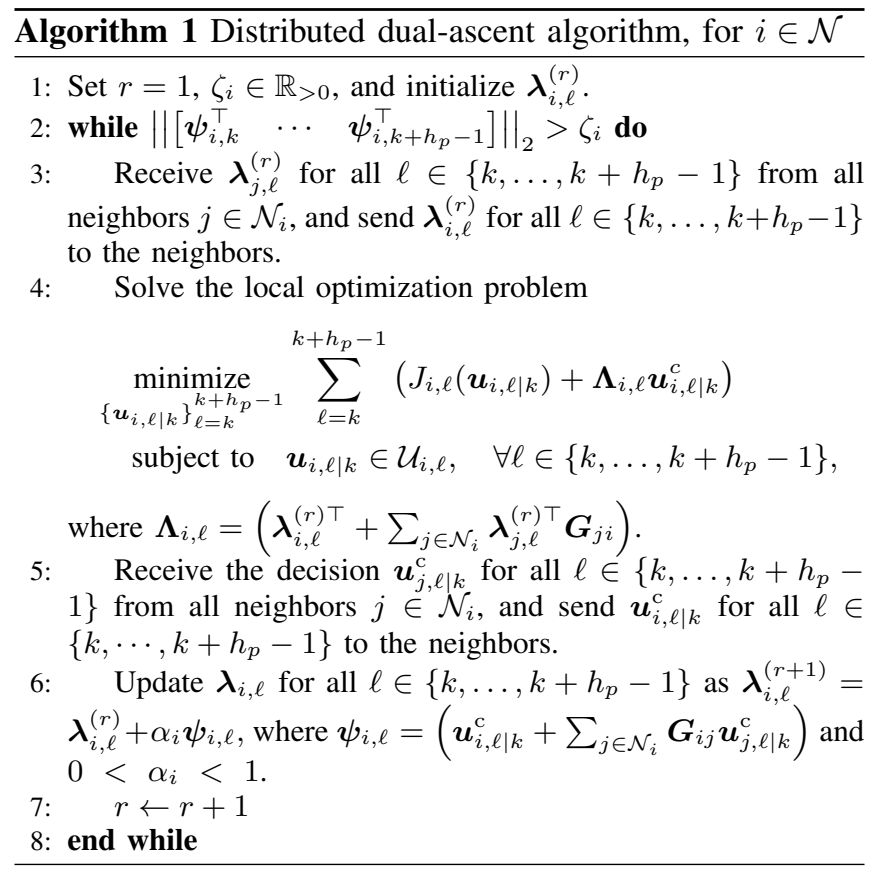

Definition 2: Given $f \in \mathbb{Z}_{\geq 1}$, the set of adversarial agents is $f$-local if $\left|\mathcal{A} \cap \mathcal{N}_{i}\right| \leq f$, for all $i \in \mathcal{N}$.

Assumption 1: Each agent $i \in \mathcal{N}$ has at most one adversarial neighbor, i.e., $f=1$.

Definition 3: An attack is an event at which an adversarial agent $i \in \mathcal{A}$ implements a control input that is different than the decision obtained from the distributed strategy, i.e., $\boldsymbol{u}_{i, k} \neq \boldsymbol{u}_{i, k \mid k}^{\star}$ for some $k$.

Assumption 2: The probability of attack, for each $i \in \mathcal{A}$, is lower bounded by a positive scalar denoted by $p_{i}^{\mathrm{a}}>0$. $\square$

The case of $f=1$ imposed by Assumption 1 is indeed restrictive and the more general case is left for future studies. By performing an attack defined in Definition 3, an adversarial agent might gain benefit from its neighbors. For instance, an adversarial agent may produce an energy quantity smaller than the amount that has been decided from the distributed algorithm. It then asks its neighbors to compensate the deficiency of power. This attack is possible since these agents are connected and the power balance equations must be met.

\section{Two-SteP SCENARIO-BASED RobUSTIFICATION}

Since the power disturbances of each microgrid, $p_{i, \ell}^{\mathrm{d}}$, for all $\ell \in\left\{k, \ldots, k+h_{p}-1\right\}$, cannot be known in advance, we can only consider the forecast of $p_{i, \ell}^{\mathrm{d}}$, denoted by $\hat{p}_{i, \ell}^{\mathrm{d}}$, and assume the difference between the forecast and the actual value as an uncertain variable denoted by $w_{i, k}^{\mathrm{d}} \in \mathbb{R}$, i.e.,

$$
w_{i, k}^{\mathrm{d}}=p_{i, k}^{\mathrm{d}}-\hat{p}_{i, k}^{\mathrm{d}} .
$$

Similarly, an attack can also be considered as another source of uncertainty, which is denoted by $w_{i, k}^{\mathrm{a}} \in \mathbb{R}$. Hence, the uncertainties of microgrid $i$ can be denoted by $\boldsymbol{w}_{i, k}=$ $\left[w_{i, k}^{\mathrm{d}} w_{i, k}^{\mathrm{a}}\right]^{\top}$ and we assume that this uncertainty is a random process as follows.
Assumption 3: Let $\Omega_{i} \subseteq \mathbb{R}^{2}$, for each $i \in \mathcal{N}$, be an uncertain set bounded and endowed with a Borel $\sigma$-algebra. For each microgrid $i \in \mathcal{N}, \boldsymbol{w}_{i, k} \in \Omega_{i}$, is an independent and identically distributed (i.i.d) random process.

The energy storage units can help to deal with the uncertain disturbance. In order to take into account this strategy and the disturbance, the formulation of the dynamic economic dispatch problem in Section II-A is adjusted as follows. Let $\hat{p}_{i, k}^{\text {st }}$ denote the nominal power delivered to/from the storage unit and consider that

$$
p_{i, k}^{\mathrm{st}}=\hat{p}_{i, k}^{\mathrm{st}}+\mathbb{1}^{\top} \boldsymbol{w}_{i, k}, \quad \forall i \in \mathcal{N}
$$

By introducing $w_{i, k}^{\mathrm{a}}$ in (5) and from (8) and (9), we obtain the local power balance equation as follows:

$$
\hat{p}_{i, k}^{\mathrm{d}}-\hat{p}_{i, k}^{\mathrm{st}}-p_{i, k}^{\mathrm{g}}-p_{i, k}^{\mathrm{im}}-\sum_{j \in \mathcal{N}_{i}} p_{j i, k}^{\mathrm{t}}=0 .
$$

By considering (9) as additional constraints, as well as (10) instead of (5), we specify how the disturbance affects the system. In particular, it can be seen that the satisfaction of constraints (2) now also depends on the disturbance.

Furthermore, we reformulate Problem (7) as a chanceconstrained problem as follows:

$$
\begin{aligned}
& \underset{\left\{\left\{\boldsymbol{u}_{i, \ell \mid k}\right\}_{i \in \mathcal{N}}\right\}_{\ell=k}^{k+h_{p}-1}}{\operatorname{minimize}} \sum_{i \in \mathcal{N}} \sum_{\ell=k}^{k+h_{p}-1} J_{i, \ell}\left(\boldsymbol{u}_{i, \ell \mid k}\right) \\
& \quad \text { subject to }(7 \mathrm{c}), \forall \ell \in\left\{k, \ldots, k+h_{p}-1\right\}, \\
& \mathbb{P}\left(\boldsymbol{F}_{i} \boldsymbol{u}_{i, \ell \mid k}+\boldsymbol{F}_{\mathrm{w}, i} \boldsymbol{w}_{i, \ell} \leq \boldsymbol{f}_{i, \ell} \mid \boldsymbol{w}_{i, \ell} \in \Omega_{i},\right. \\
& \left.\forall \ell \in\left\{k, \ldots, k+h_{p}-1\right\}\right) \geq 1-\varepsilon_{i}, \forall i \in \mathcal{N},
\end{aligned}
$$

where $\boldsymbol{u}_{i}=\left[\begin{array}{llll}\hat{p}_{i, k}^{\mathrm{st}} & p_{i, k}^{\mathrm{g}} & p_{i, k}^{\mathrm{im}} & \boldsymbol{u}_{i, k}^{\mathrm{c} \top}\end{array}\right]^{\top} \in \mathbb{R}^{3+\left|\mathcal{N}_{i}\right|}$; the inequality $\boldsymbol{F}_{i} \boldsymbol{u}_{i, \ell \mid k}+\boldsymbol{F}_{\mathrm{w}, i} \boldsymbol{w}_{i, \ell} \leq \boldsymbol{f}_{i, \ell}$ is a compact form of the local constraints (1)-(4), (9), and (10), with appropriate $\boldsymbol{F}_{i}$ and $\boldsymbol{F}_{\mathrm{w}, i}$ matrices and $\boldsymbol{f}_{i, \ell}$ vector; and $\varepsilon_{i} \in(0,1)$ is the maximum violation level. Any solution of Problem (11) is referred to as an $\varepsilon$-level solution, where $\varepsilon=\sum_{i \in \mathcal{N}} \varepsilon_{i}$ and we follow a stochastic approach [7] to compute such a solution.

\section{A. Computing Probabilistic Bounds}

In this step, we solve a randomized program to compute a set that probabilistically bounds the uncertainty of the chance-constrained problem. Since in Problem (11), each microgrid has a sequence of uncertain variables, i.e., $\boldsymbol{w}_{i, \ell}$, for all $\ell \in\left\{k, \ldots, k+h_{p}-1\right\}$, let the set that bounds a portion of the probability mass of $\left[\boldsymbol{w}_{i, k}^{\top} \cdots \boldsymbol{w}_{i, k+h_{p}-1}^{\top}\right]^{\top}$ be denoted by $\mathcal{B}_{i, k}^{\star}$. We define $\mathcal{B}_{i, k}^{\star}$ to be a polyhedral set, i.e., $\mathcal{B}_{i, k}^{\star}=\prod_{\ell=k}^{k+h_{p}-1}\left\{\boldsymbol{\tau} \in \mathbb{R}^{2}: \underline{\boldsymbol{\tau}}_{i, \ell \mid k}^{\star} \leq \boldsymbol{\tau} \leq \overline{\boldsymbol{\tau}}_{i, \ell \mid k}^{\star}\right\}$, where $\underline{\boldsymbol{\tau}}_{i, \ell \mid k}^{\star}, \overline{\boldsymbol{\tau}}_{i, \ell \mid k}^{\star} \in \mathbb{R}^{2}$ denote the lower and upper bounds of $\boldsymbol{w}_{i, \ell}$, respectively, and consist of two components since there are two sources of uncertainty.

In order to compute $\mathcal{B}_{i, k}^{\star}$, we follow a probabilistic approach based on the so-called scenario approach of [7]. In particular, this is done by first posing a chance-constrained problem to obtain the probabilistic bounds and then approximating the solution of that problem by solving an 
optimization based on sampling in the uncertain set as

$$
\underset{\left\{\underline{\boldsymbol{\tau}}_{i, \ell \mid k}, \overline{\boldsymbol{\tau}}_{i, \ell \mid k}\right\}_{\ell=k}^{k+h_{p}-1}}{\operatorname{minimize}} \sum_{\ell=k}^{k+h_{p}-1} \mathbb{1}^{\top}\left(\overline{\boldsymbol{\tau}}_{i, \ell \mid k}-\underline{\boldsymbol{\tau}}_{i, \ell \mid k}\right)
$$

subject to

$$
\boldsymbol{w}_{i, \ell}^{(s)} \in\left[\underline{\boldsymbol{\tau}}_{i, \ell \mid k}, \overline{\boldsymbol{\tau}}_{i, \ell \mid k}\right], \quad s=1, \ldots, n_{s, i},
$$

for all $\ell \in\left\{k, \ldots, k+h_{p}-1\right\}$, where $\boldsymbol{w}_{i, \ell}^{(s)}$ denote a scenario of $\boldsymbol{w}_{i, \ell}$, generated according to the probability measure for $\boldsymbol{w}_{i, \ell}$ in an i.i.d. manner, and $n_{s, i}$ is the number of scenarios. According to [7], for the desired level of confidence $\beta_{i} \in$ $(0,1)$, we must choose $n_{s, i}$ satisfying

$$
n_{s, i} \geq \frac{e}{\varepsilon_{i}(e-1)}\left(4 h_{p}-1+\ln \frac{1}{\beta_{i}}\right) .
$$

With some abuse of notation, let the set $\mathcal{B}_{i, k}^{\star}$ be constructed from the solution of Problem (12). This set is a solution of the chance-constrained problem to obtain the bounds with probability at least $1-\beta_{i}$.

\section{B. Robust Reformulation}

Based on the bounds $\mathcal{B}_{i, k}^{\star}$, for all $i \in \mathcal{N}$, we formulate a robust counterpart of Problem (11). Since the local constraints are convex, we can apply the vertex enumeration method, i.e., substituting the uncertain variable $\boldsymbol{w}_{i, \ell}$ with the vertices of $\mathcal{B}_{i, k}^{\star}$ [7]. The robust formulation associated to Problem (11) is stated as follows:

$$
\begin{aligned}
& \underset{\left\{\left\{\boldsymbol{u}_{i, \ell \mid k}\right\}_{i \in \mathcal{N}}\right\}_{\ell=k}^{k+h_{p}-1}}{\operatorname{minimize}} \sum_{i \in \mathcal{N}} \sum_{\ell=k}^{k+h_{p}-1} J_{i, \ell}\left(\boldsymbol{u}_{i, \ell \mid k}\right) \\
& \text { subject to }(7 \mathrm{c}), \\
& \boldsymbol{F}_{i} \boldsymbol{u}_{i, \ell \mid k}+\boldsymbol{F}_{\mathrm{w}, i} \boldsymbol{w}_{i, \ell \mid k} \leq \boldsymbol{f}_{i, \ell}, \\
& \boldsymbol{w}_{i, \ell \mid k} \in \mathcal{V}\left(\mathcal{B}_{i, k}^{\star}, \ell\right),
\end{aligned}
$$

for all $\ell \in\left\{k, \ldots, k+h_{p}-1\right\}$ and $i \in \mathcal{N}$, where $\mathcal{V}\left(\mathcal{B}_{i, k}^{\star}, \ell\right)=\left\{\underline{\boldsymbol{\tau}}_{i, \ell \mid k}^{\star}, \overline{\boldsymbol{\tau}}_{i, \ell \mid k}^{\star}\right\}$ is the vertex set computed in the previous step (Section III-A). Problem (14) is convex, with a strictly convex cost function and local non-empty polyhedral constraint sets. Therefore, we can obtain the optimal solution of Problem (14) using Algorithm 1, where $\mathcal{U}_{i, k}$ is defined by constraints (14b) and (14c).

\section{Attack Identification And Mitigation}

Now, we present the attack identification and mitigation method, which is based on [6]. However, since we do not have the exact bounds of the uncertain variables, we use the probabilistic bounds obtained from Section III-A.

\section{A. Attack Detection}

Firstly, a regular agent must be able to detect an attack. To this end, a regular agent uses its SoC information to compute the total disturbance, denoted by $\delta_{i, k}$, as follows:

$$
\delta_{i, k}=x_{i, k+1}-\left(x_{i, k}+\boldsymbol{b}_{i}^{\top} \boldsymbol{u}_{i, k \mid k}\right), \quad \forall k \in \mathbb{Z}_{\geq 0},
$$

where $\boldsymbol{b}_{i}=\left[\begin{array}{ll}b_{i} & \mathbf{0}_{2+\left|\mathcal{N}_{i}\right|}^{\top}\end{array}\right]^{\top}$. By definition, $\delta_{i, k}=b_{i} \mathbb{1}^{\top} \boldsymbol{w}_{i, k}$. The probabilistic bound $\mathcal{B}_{i, k}^{\star}$ can now be used as the threshold to define whether an attack occurs. Recall that $\underline{\tau}_{i, k \mid k}^{\star}=$ $\left[\underline{\tau}_{i, k \mid k}^{\mathrm{d} \star} \quad \underline{\tau}_{i, k \mid k}^{\mathrm{a} \star}\right]^{\top}$, where $\underline{\tau}_{i, k \mid k}^{\mathrm{d} \star}$ and $\underline{\tau}_{i, k \mid k}^{\mathrm{a} \star}$ denote the lower bounds of the disturbance associated to the load and to an attack, respectively, and similarly $\bar{\tau}_{i, k \mid k}^{\star}=\left[\bar{\tau}_{i, k \mid k}^{\mathrm{d} \star} \bar{\tau}_{i, k \mid k}^{\mathrm{a} \star}\right]^{\top}$, where $\bar{\tau}_{i, k \mid k}^{\mathrm{d} \star}$ and $\bar{\tau}_{i, k \mid k}^{\mathrm{a} \star}$ denote the corresponding upper bounds. Then, the attack detection is defined as follows.

Definition 4: For each regular agent $i \in \mathcal{R}$ and $k \in \mathbb{Z}_{\geq 0}$, let $d_{i, k}^{\mathrm{a}} \in\{0,1\}$ be the indicator that detects attacks. If

$$
\underline{\tau}_{i, k \mid k}^{\mathrm{d} \star} \leq \delta_{i, k} / b_{i} \leq \bar{\tau}_{i, k \mid k}^{\mathrm{d} \star}
$$

then there is no attack detected by agent $i$ and $d_{i, k}^{\mathrm{a}}=0$. Otherwise, $d_{i, k}^{\mathrm{a}}=1$, implying an attack is detected.

For this attack detection mechanism, we further introduce the notations of detectable attacks and false attack detections.

Definition 5: An attack is detectable if $w_{i, k}^{\mathrm{a}} \neq 0$ such that (16) does not hold. On the other hand, an attack is undetectable if $w_{i, k}^{\mathrm{a}} \neq 0$ such that (16) holds.

Assumption 4: The probability of the undetectable attacks received by agent $i \in \mathcal{R}$, denoted by $p_{i}^{\text {ua }}$, is less than 1 .

Definition 6: A false attack detection occurs if $w_{i, k}^{\mathrm{d}} \leq$ $\underline{\tau}_{i, k \mid k}^{\mathrm{d} \star}$ or $w_{i, k}^{\mathrm{d}} \geq \bar{\tau}_{i, k \mid k}^{\mathrm{d} \star}$ when $w_{i, k}^{\mathrm{a}}=0$.

\section{B. Identification and Mitigation Method}

We suppose that regular agents do not have prior knowledge of the occurrence of the attacks, but they have an initial expectation on the probability of attacks, denoted by $\hat{p}_{i}^{\mathrm{a}} \in(0,1)$, for each $i \in \mathcal{R}$.

Each agent, $i \in \mathcal{R}$, considers the set of hypotheses given by $\mathcal{H}_{i}=\left\{\mathbf{H}_{i}^{0}, \mathbf{H}_{i}^{j}: j \in \mathcal{N}_{i}\right\}$, where the hypotheses are defined as follows:

- $\mathbf{H}_{i}^{0}$ : There is no adversarial agent,

- $\mathbf{H}_{i}^{j}$ : Neighbor $j$ is an adversarial agent,

for all $j \in \mathcal{N}_{i}$. The Bayesian inference is used as the model to update the probability of the hypotheses as follows:

$$
\mathbb{P}_{k+1}\left(\mathbf{H}_{i}^{j}\right)=\frac{\mathbb{P}_{k}\left(\mathbf{H}_{i}^{j}\right) \mathbb{P}_{k}\left(d_{i, k}^{\mathrm{a}} \mid \mathbf{H}_{i}^{j}\right)}{\mathbb{P}_{k}\left(d_{i, k}^{\mathrm{a}}\right)},
$$

for all $\mathbf{H}_{i}^{j} \in \mathcal{H}_{i}$, where $\mathbb{P}_{k+1}\left(\mathbf{H}_{i}^{j}\right)$ is the a posteriori probability of $\mathbf{H}_{i}^{j}$ given the event $d_{i, k}^{\text {a }}$, i.e., $\mathbb{P}_{k+1}\left(\mathbf{H}_{i}^{j}\right)=$ $\mathbb{P}\left(\mathbf{H}_{i}^{j} \mid d_{i, k}^{\mathrm{a}}\right) ; \mathbb{P}_{k}\left(\mathbf{H}_{i}^{j}\right)$ denotes the probability of hypothesis $\mathbf{H}_{i}^{j}$ at time instant $k ; \mathbb{P}_{k}\left(d_{i, k}^{\mathrm{a}}\right)$ denotes the marginal likelihood of $d_{i, k}^{\mathrm{a}}$; and $\mathbb{P}_{k}\left(d_{i, k}^{\mathrm{a}} \mid \mathbf{H}_{i}^{j}\right)$ denotes the probability of observing $d_{i, k}^{\mathrm{a}}$ given hypothesis $\mathbf{H}_{i}^{j}$. The probability $\mathbb{P}_{k}\left(d_{i, k}^{\mathrm{a}} \mid \mathbf{H}_{i}^{j}\right)$ is formulated as follows:

$$
\begin{aligned}
& \mathbb{P}_{k}\left(d_{i, k}^{\mathrm{a}}=0 \mid \mathbf{H}_{i}^{j}\right)= \begin{cases}1, & \text { for } j=0, \\
1-v_{i, k}^{j} \hat{p}_{i}^{\mathrm{a}}, & \text { for all } j \in \mathcal{N}_{i},\end{cases} \\
& \mathbb{P}_{k}\left(d_{i, k}^{\mathrm{a}}=1 \mid \mathbf{H}_{i}^{j}\right)= \begin{cases}0, & \text { for } j=0, \\
v_{i, k}^{j} \hat{p}_{i}^{\mathrm{a}}, & \text { for all } j \in \mathcal{N}_{i},\end{cases}
\end{aligned}
$$

where $v_{i, k}^{j} \in\{0,1\}$, denotes the decision whether agent $i$ connects to and negotiates with neighbor $j$, i.e., $v_{i, k}^{j}=1$ if agent $i$ connects to neighbor $j$, whereas $v_{i, k}^{j}=0$ otherwise. The probabilities of all hypotheses are initialized as follows: $\mathbb{P}_{0}\left(\mathbf{H}_{i}^{j}\right)=\hat{p}_{i}^{\mathrm{a}} /\left|\mathcal{N}_{i}\right|$, for all $j \in \mathcal{N}_{i}$, and $\mathbb{P}_{0}\left(\mathbf{H}_{i}^{0}\right)=1-\hat{p}_{i}^{\mathrm{a}}$, implying that it is initially considered that each neighbor is 


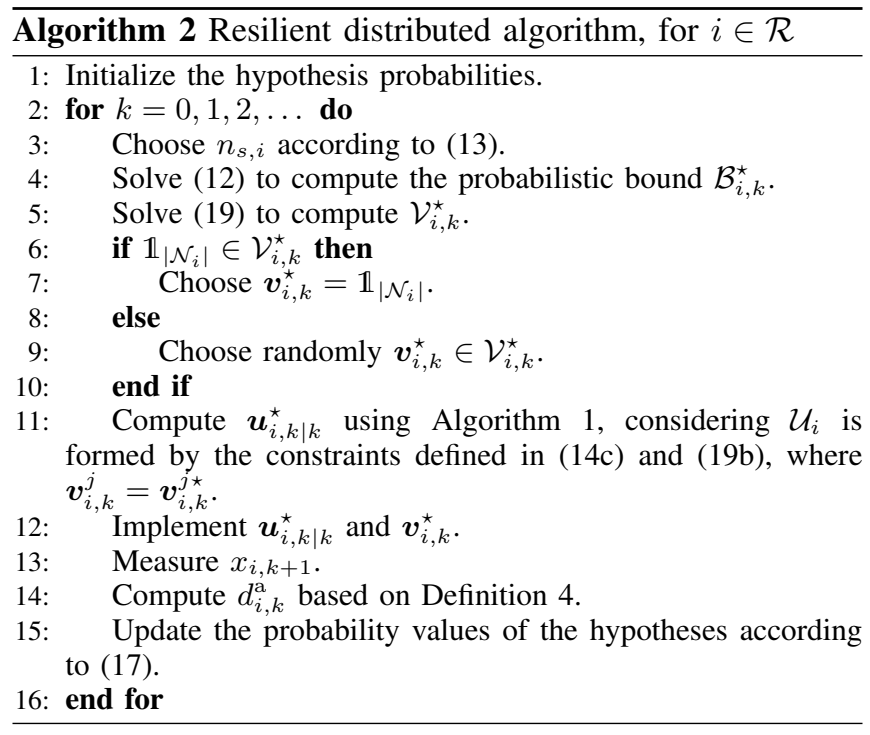

equally likely to be adversarial. Note that $\hat{p}_{i}^{\mathrm{a}}$ does not need to be equal to $p_{j}^{\mathrm{a}}$, for $j \in \mathcal{N}_{i} \cap \mathcal{A}$.

In order to compute $v_{i, k}^{j}$, for all $j \in \mathcal{N}_{i}$, agent $i$ solves a local mixed-integer optimization problem as follows:

$$
\begin{aligned}
& \underset{\substack{\boldsymbol{v}_{i, k},\left\{\boldsymbol{u}_{i, \ell \mid k}\right\}_{\ell=k}^{k+h_{p}-1} \\
\text { subject to }(14 \mathrm{c}),}}{\operatorname{minimize}} \sum_{\ell=k}^{k+h_{p}-1} J_{i, \ell}\left(\boldsymbol{u}_{i, \ell \mid k}\right)+J_{i}^{\mathrm{v}}\left(\boldsymbol{v}_{i, k}\right) \\
& \boldsymbol{F}_{i} \boldsymbol{u}_{i, \ell \mid k}+\boldsymbol{F}_{\mathrm{w}, i} \boldsymbol{w}_{i, \ell \mid k}+\boldsymbol{F}_{\mathrm{v}, i} \boldsymbol{v}_{i, k} \leq \boldsymbol{f}_{i, \ell}^{\mathrm{lc}}, \\
& \boldsymbol{v}_{i, k} \in\left\{\boldsymbol{v} \in\{0,1\}^{\left|\mathcal{N}_{i}\right|}: \mathbb{1}^{\top} \boldsymbol{v} \geq\left|\mathcal{N}_{i}\right|-1\right\},
\end{aligned}
$$

for all $\ell \in\left\{k, \ldots, k+h_{p}-1\right\}$, where $\boldsymbol{v}_{i, k}=\left[v_{i, k}^{j}\right]_{j \in \mathcal{N}_{i}}^{\top} \in$ $\{0,1\}^{\left|\mathcal{N}_{i}\right|}$. The cost function $J_{i}^{\mathrm{v}}\left(\boldsymbol{v}_{i, k}\right): \mathbb{R}^{\left|\mathcal{N}_{i}\right|} \rightarrow \mathbb{R}$ penalizes the decision of having a connection with the neighbors, i.e., $J_{i}^{\mathrm{v}}\left(\boldsymbol{v}_{i, k}\right)=\gamma_{i} n_{i, k}^{\mathrm{a}} \sum_{j \in \mathcal{N}_{i}} \mathbb{P}_{k}\left(\mathbf{H}_{i}^{j}\right) v_{i, k}^{j}$, where $\gamma_{i} \in \mathbb{R}_{>0}$ denotes a weight that must be predefined and $n_{i, k}^{\mathrm{a}}$ denotes the number of attacks that agent $i$ has detected, i.e., $n_{i, k}^{\mathrm{a}}=$ $\sum_{\ell=0}^{k} d_{i, \ell}^{\text {at }}$. Moreover, (19b) is obtained from (1)-(3), (9), (10) and from the following expression:

$$
-p_{i}^{\mathrm{t}, \max } v_{i, k}^{j} \leq p_{j i, \ell}^{\mathrm{t}} \leq p_{i}^{\mathrm{t}, \max } v_{i, k}^{j}, \quad \forall j \in \mathcal{N}_{i},
$$

for all $\ell \in\left\{k, \ldots, k+h_{p}-1\right\}$, whereas, the constraint (19c) implies that agent $i$ can only disconnect from at most one neighbor. Finally, let $\mathcal{V}_{i, k}^{\star} \in\{0,1\}^{\left|\mathcal{N}_{i}\right|}$ be the set of minimizers of Problem (19) and suppose that $\boldsymbol{v}_{i, k}^{\star}=\left[v_{i, k}^{j \star}\right]_{j \in \mathcal{N}_{i}}^{\top}$ is chosen from $\mathcal{V}_{i, k}^{\star}$, i.e., $\boldsymbol{v}_{i, k}^{\star} \in \mathcal{V}_{i, k}^{\star}$. Hence, in order to include the connection decision in the robust problem (14), the local constraints (14b) are switched by (19b) with $\boldsymbol{v}_{i, k}=\boldsymbol{v}_{i, k}^{\star}$, for all $i \in \mathcal{N}$.

\section{Analysis of the Overall Method}

The overall method is summarized in Algorithm 2. Related to the part of implementing $\boldsymbol{v}_{i, k}^{\star}$, we consider that any agent can temporarily disconnect the physical links between itself and its neighbors, respecting the decision of $\boldsymbol{v}_{i, k}^{\star}$. This fact implies that two agents, $i$ and $j$, where $(i, j) \in \mathcal{E}$, can only exchange energy if and only if $v_{i, k}^{j \star}=v_{j, k}^{i \star}=1$. Now, we provide some analysis results of the algorithm, in terms of the characteristics of the obtained solution (Proposition 1) and the result of the identification method (Proposition 2). Prior to stating these results, we introduce Assumption 5.

Assumption 5: The feasible set of Problem (14) is nonempty. Moreover, this set contains a subset in which $p_{i j, \ell}^{\mathrm{t}}=$ $p_{j i, \ell}^{\mathrm{t}}=0$, for all $\ell \in\left\{k, \ldots, k+h_{p}-1\right\}$, and $(i, j) \in \mathcal{E}$. $\square$

The existence of a nonempty feasible region in Problem (14) depends on the scenario realization, which determines the construction of the probabilistic bounds. Therefore, each agent can compute the bounds such that Assumption 5 holds. Furthermore, the second part of Assumption 5 implies that each microgrid can operate in the island mode.

Proposition 1: Suppose that Assumptions 3 and 5 hold. Furthermore, suppose that each agent $i \in \mathcal{N}$ applies steps 4, 5-11 of Algorithm 2 to compute $\boldsymbol{u}_{i, k \mid k}^{\star}$. Then, the implemented decision, $\boldsymbol{u}_{i, k \mid k}^{\star}$, for each $i \in \mathcal{R}$, is an $\varepsilon$-level solution of Problem (11), with level of inexactness $\zeta_{i}$ and probability at least $1-\beta$, where $\varepsilon=\sum_{i \in \mathcal{N}} \varepsilon_{i}$ and $\beta=\sum_{i \in \mathcal{N}} \beta_{i}$.

Proof: Due to the space constraint, we outline the main ideas of the proof. The feasible set of the problem solved in step 11 of Algorithm 2 is a subset of feasible solutions of Problem (14). Then, the characteristics follow from the results of implementing Algorithm 1 and the stochastic method explained in Section III.

Proposition 2: Suppose that Assumptions 1-5 hold, a regular agent $i \in \mathcal{R}$ applies Algorithm 2, and there exists an adversarial neighbor of agent $i$. If there is no false detection, then agent $i$ correctly identifies the adversarial neighbor.

Proof: Due to the space limitation, we provide a sketch of the proof. We evaluate the evolution of each hypothesis probability $\mathbb{P}_{k}\left(\mathbf{H}_{i}^{j}\right)$ based on (17), particularly when a regular agent $i$ keeps blocking one of the neighbors. Once the adversarial neighbor is blocked, it can be shown that it will always be blocked at the later time instants and the convergence of each hypothesis probability can be analytically shown. On the other hand, when a regular neighbor is blocked, the agent will receive an attack and it will later choose to block a different neighbor, until the adversarial agent is blocked. Hence, only the hypothesis probability associated to the adversarial neighbor eventually converges to 1 .

\section{CASE STUdy}

We consider the PG\&E 69-bus distribution network with additional dispatchable generation, solar-based power generation, and storage units. The network is partitioned into eight microgrids (agents) [9]. The topology of this interconnectedmicrogrid system is shown in Fig. 1. Furthermore, the parameter values of the components of each microgrid are given in Table I. We suppose that microgrids 1, 2, 5, and 6 have industrial load profiles whereas the others have residential profiles. The load and solar-based generation profiles are based on realistic data [10], [11]. In addition, we consider that agents 2, 6, and 7 are adversarial and they attack with $p_{i}^{\mathrm{a}}=0.3$. The attack strategy of the adversarial agents is to reduce the production of their dispatchable generation units randomly. It is set that the simulation time is one day (96 


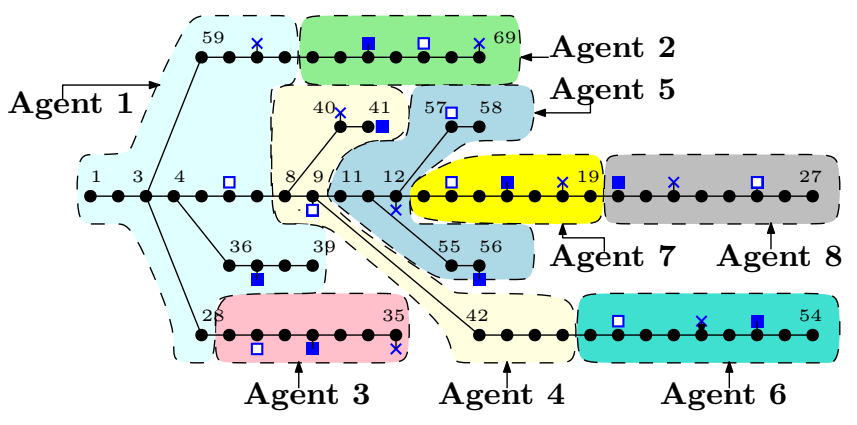

Fig. 1. The PG\&E 69-bus distribution network [9]. Squares indicate the distributed generation units, i.e., $\mathbf{\square}$ and $\square$ represent a renewable generation unit and a dispatchabale generator, respectively, whereas crosses, $\times$, indicate the storages.

TABLE I

PARAMETERS OF THE MiCROGRIDS

\begin{tabular}{cccc}
\hline Parameters & Value & Unit & Agent \\
\hline$x_{i}^{\mathrm{min}}, x_{i}^{\mathrm{max}}, x_{i, 0}$ & $30,80,50$ & $\%$ & all \\
\hline$p_{i}^{\mathrm{ch}}, p_{i}^{\mathrm{dh}}$ & 300,300 & $\mathrm{~kW}$ & all \\
\hline$p_{i}^{\mathrm{g}, \min }, p_{i}^{\mathrm{g}, \max }$ & 0,1000 & $\mathrm{~kW}$ & $3,4,7,8$ \\
& 0,2000 & $\mathrm{~kW}$ & $1,2,5,6$ \\
\hline$p_{i}^{\mathrm{t}, \max }, p_{i}^{\mathrm{im}, \max }$ & 110,2000 & $\mathrm{~kW}$ & all \\
\hline$e_{\mathrm{cap}, i}$ & 500 & $\mathrm{kWh}$ & $3,4,7,8$ \\
& 1000 & $\mathrm{kWh}$ & $1,2,5,6$ \\
\hline$c_{i}^{\mathrm{st}}, c_{i}^{\mathrm{im}}, c_{i}^{\mathrm{t}}$ & $1,250,0.1$ & - & all \\
\hline$c_{i}^{\mathrm{g}}$ & 5 & - & $2,4,6,7$ \\
& 10 & - & $1,3,5,8$ \\
\hline$a_{i}, \varepsilon_{i}, \beta_{i}$ & $0.98,0.01,0.05$ & - & all \\
\hline
\end{tabular}

steps), $T_{s}=15$ minutes, $h_{p}=4$, and $\zeta_{i}=5$, for all $i \in \mathcal{N}$. Low-level local controllers are placed at each microgrid that control the dispatchable generation and storage units such that the set points computed are met. Figs. 2 and 3 show some plots of the simulation results. From the top plot of Fig. 2, it is observed that the SoC value of agent 1 stays in the limit for all time steps, showing the robustness of the decisions with respect to the attacks and system disturbance. Fig. 3 shows how agent 1 manages to identify and disconnect from its adversarial neighbor (agent 2) using Algorithm 2.

\section{CONCLUSiOn AND Future Work}

A combination of passive and active schemes to deal with non-compliance in a distributed energy management of interconnected microgrid systems has been presented and analyzed. The passive scheme uses a two-step stochastic method to robustify the decisions against the uncertain at-

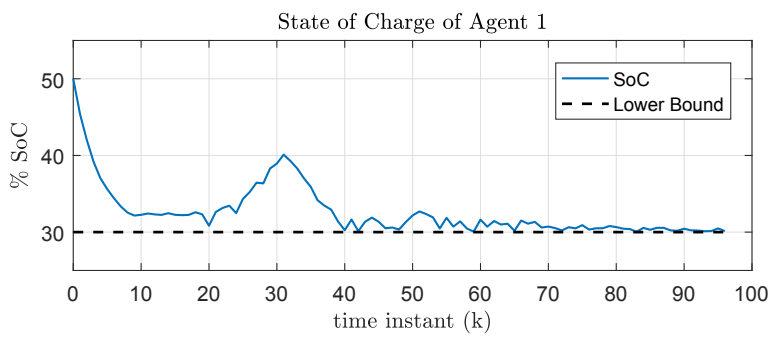

Fig. 2. The evolution of $\mathrm{SoC}$ of agent 1.

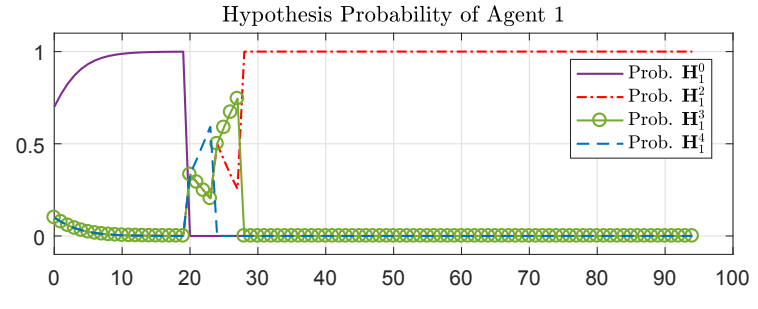

Connection Decision of Agent 1

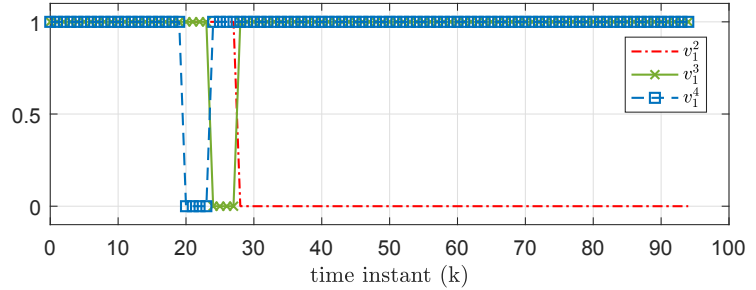

Fig. 3. The evolution of each hypothesis probability of agent 1 (top plot) and the connection decision of agent 1 at each time instant (bottom plot).

tacks and system disturbances. On the other hand, the active scheme employs a hypothesis testing method to identify the adversarial agents. As an ongoing work, the proposed method is extended for more general cases, i.e., $f \geq 1$, and attacks that are hard to detect.

\section{REFERENCES}

[1] C. A. Hans, P. Braun, J. Raisch, L. Grune, and C. Reincke-Collon, "Hierarchical distributed model predictive control of interconnected microgrids," IEEE Trans. Sustainable Energy, vol. 10, no. 1, pp. 407416, 2019.

[2] K. Baker, J. Guo, G. Hug, and X. Li, "Distributed MPC for efficient coordination of storage and renewable energy sources across contro areas," IEEE Transactions on Smart Grid, vol. 7, no. 2, pp. 992-1001, 2016.

[3] G. Hug, S. Kar, and C. Wu, "Consensus + innovations approach for distributed multiagent coordination in a microgrid," IEEE Transactions on Smart Grid, vol. 6, no. 4, pp. 1893-1903, 2015.

[4] D. K. Molzahn, F. Dörfler, H. Sandberg, S. H. Low, S. Chakrabarti, R. Baldick, and J. Lavaei, "A survey of distributed optimization and control algorithms for electric power systems," IEEE Transactions on Smart Grid, vol. 8, no. 6, pp. 2941-2962, 2017.

[5] T. Morstyn, B. Hredzak, and V. G. Agelidis, "Control strategies for microgrids with distributed energy storage systems: An overview," IEEE Transactions on Smart Grid, vol. 9, no. 4, pp. 3652-3666, 2018.

[6] W. Ananduta, J. M. Maestre, C. Ocampo-Martinez, and H. Ishii, "Resilient distributed energy management for systems of interconnected microgrids," in Proceedings of the IEEE 57th Conference on Decision and Control (CDC), Miami, USA, 2018, pp. 3159-3164.

[7] K. Margellos, P. Goulart, and J. Lygeros, "On the road between robust optimization and the scenario approach for chance constrained optimization problems," IEEE Transactions on Automatic Control, vol. 59 , no. 8 , pp. 2258-2263, 2014.

[8] S. Boyd and L. Vandenberghe, Convex Optimization. Cambridge University Press, 2010.

[9] S. A. Arefifar, Y. A. R. I. Mohamed, and T. H. M. El-Fouly, "Supply-adequacy-based optimal construction of microgrids in smart distribution systems," IEEE Transactions on Smart Grid, vol. 3, no. 3 , pp. 1491-1502, 2012.

[10] Y. Hayashi, Y. Fujimoto, H. Ishii, Y. Takenobu, H. Kikusato, S. Yoshizawa, Y. Amano, S. I. Tanabe, Y. Yamaguchi, Y. Shimoda, J. Yoshinaga, M. Watanabe, S. Sasaki, T. Koike, H. A. Jacobsen, and K. Tomsovic, "Versatile modeling platform for cooperative energy management systems in smart cities," Proceedings of the IEEE, vol. 106, no. 4, pp. 594-612, 2018

[11] New Energy Industrial Technology Development Organization (NEDO). (2002-2007) Demonstrative research on grid-interconnection of clustered photovoltaic power generation systems. 